\title{
Effects of PVA-PEG binders system on microstructure and properties of sintered alumina
}

\begin{abstract}
In this work, the effects of binder compositions on the mechanical properties, density, volume shrinkage and microstructure of sintered alumina were investigated. Three different formulations of alumina samples were prepared by mixing alumina with $3 \%$ of poly (ethylene glycol) (PEG) with 1 to $3 \mathrm{wt} \%$ of poly (vinyl alcohol) (PVA) binders. The degradation temperature of binder was investigated by means of thermogravimetry analysis (TGA). Diametral compressive test was conducted to determine the tensile strength of sintered alumina. Scanning electron microscopy (SEM) was used to characterise the microstructure and fracture surface analysis. It was found that PVA binder plays important roles in sintering of alumina. The relative density and tensile strength increase as the percentages of PVA increase for each sintering temperature but the volume shrinkages are not much affected by binder composition. The results inferred that alumina samples which have 3\%wtPVA-3\%wtPEG binder sintered at $1600{ }^{\circ} \mathrm{C}$ shows the highest tensile strength (129 $\mathrm{MPa})$ compared to other formulations.
\end{abstract}

Keyword: Alumina; Binder; Polyethylene glycol (PEG); Polyvinyl alcohol (PVA) 